九州大学学術情報リポジトリ

Kyushu University Institutional Repository

\title{
A Multivariate Analogue of Pooling of Data
}

Sato, Sokuro

Saga University

https://doi.org/10.5109/13007

出版情報: 統計数理研究. 10 (3/4)，pp.61-76，1962-10. Research Association of Statistical Sciences

バージョン：

権利関係 : 


\title{
A Multivariate analogue of pooling OF DATA
}

\author{
By \\ Sokuro S Sto \\ (Received August 16th, 1961)
}

\section{Summary and introduction}

In this paper the author attempts to extend the inferences of a $k$ dimensional mean vector on the basis of pooling data in a multivariate normal case and to give concretely certain formulae and properties of them.

The principle and some statistical methods of pooling data have been discussed by Bancroft [1], Kitagawa [1], [2], Bennet [1], Asano [1] and various authors and developed mainly in case when the observations were obtained from the univariate populations. Recently Asano and the author of this paper [1] dealt the inference of a mean vector and a dispersion matrix on the basis of pooling data in the bivariate case. And in their Introduction it has been noted that the inference of a mean vector may be also expressed by the similar formulae and properties in the general $k$ dimensional multivariate case.

Hence this paper may be considered to be partially an extension of the previous paper of Asano and Sato [1]. Type 1 of this paper gives us the inference of population mean vector with known population dispersion matrix and Type 2 with unknown population dispersion matrix.

In conclusion the author wishes his hearty thanks to Prof. T. Kitagawa and Mr. C. Asano for their kind suggestions and encouragement.

\section{$\S 2$. Pooling of sample mean vector}

2.1. Type 1. (The inference of population mean vector with “known" population dispersion matrix)

Let $O_{N_{1}}:\left(\boldsymbol{x}^{(1)}, \boldsymbol{x}^{(1)}, \cdots, \boldsymbol{x}_{N_{1}}^{(1)}\right)$ be a random sample of $N_{1}$ vector observations from a $k$-variate non-degenerate normal population $N\left[\mu^{(1)}, \Sigma\right]$ and let $O_{N_{2}}$ : $\left(\boldsymbol{x}^{(2)}, \boldsymbol{x}^{(2)}, \cdots, \boldsymbol{x}_{N_{2}}^{(2)}\right)$ be another random sample of $N_{2}$ from some $k$-variate normal population $N\left[\mu^{(2)}, \Sigma\right]$. The values of these two common population dispersion matrices are known to us, but the populations have not necessarily the same population mean vector. The distinction between these populations may be regarded, however, as hypothetical. Let us suppose that we may pool the two sample mean vectors and form an estimate vector 
of the assumed same population mean vectors simultaneously in case when testing a hypothesis that $\boldsymbol{\mu}^{(1)}=\boldsymbol{\mu}^{(2)}$ shows that the hypothesis cannot be rejected.

Our rule of inference procedure is as follows:

(i) Let $\overline{\boldsymbol{x}}^{(i)}$ be sample mean vector defined by $O_{v i}, \quad(i=1,2)$

(ii) Let the statistic $\boldsymbol{U}^{\prime} \boldsymbol{U}$ be defined by

$$
\boldsymbol{U}^{\prime} \boldsymbol{U}=\left(\boldsymbol{x}^{(2)}-\boldsymbol{x}^{(1)}\right)^{\prime}\left(\frac{1}{N_{1}}+\frac{1}{N_{2}^{-}}\right)^{-1} \boldsymbol{\Sigma}^{-1}\left(\boldsymbol{x}^{(2)}-\overline{\boldsymbol{x}}^{(1)}\right),
$$

where $\boldsymbol{x}^{(i)}=\sum_{j=1}^{N i} \boldsymbol{x}_{j}^{(i)} / N_{i}$.

(iii) Then let us define the statistic $\bar{x}$ in the following way.

$$
\begin{array}{llll}
\text { (a) } & \dot{\boldsymbol{x}}=\frac{N_{1} \boldsymbol{x}^{(1)}+N_{2} \boldsymbol{x}^{(2)}}{N_{1}+N_{2}}, & \text { if } & \boldsymbol{U}^{\prime} \boldsymbol{U} \leq \chi_{k}^{2}(\alpha) \\
\text { (b) } \boldsymbol{x}=\overline{\boldsymbol{x}}^{(1)}, & \text { if } & \boldsymbol{U}^{\prime} \boldsymbol{U}>\chi_{k}^{2}(\alpha),
\end{array}
$$

where $\chi_{k}^{2}(\alpha)$ means the significance value of $\chi^{2}$ distribution with a significance level $\alpha$ in case when the degrees of freedom is equal to $k$.

For the sake of convenience, throughout this paper, the notation of $\operatorname{Pr} .\{\overline{\boldsymbol{x}}<\boldsymbol{u}\}$ shows at the same time the probability that each element of the vector $\overline{\boldsymbol{x}}$ does not over the corresponding element of the vector $\boldsymbol{u}$ namely, the distribution functions of the respective elements of $x$ simultaneously.

Theorem 1.1. The distribution function of $\bar{x}$ is given by

$$
\begin{aligned}
& =\int \cdots \int \frac{1}{(2 \pi)^{k / 2}} \exp \left[-\frac{1}{2} \sum_{i=1}^{k} r_{i}^{2}\right] \prod_{i=1}^{k} d r_{i} \int \cdots \int \frac{1}{(2 \pi)^{k / 2}} \exp \left[-\frac{1}{2} \sum_{i=1}^{k} s_{i}^{2}\right] \prod_{i=1}^{k} d s_{i} \\
& r_{k}<m_{k} / l_{k k} \\
& r_{k-1}<\left(m_{k-1}-l_{k-1, k} \gamma_{k}\right) / l_{k-1, k-1} \\
& r_{k}<\left(m_{1}-\sum_{j=2}^{k} l_{1} r_{j}\right) / l_{11} \\
& +\int \cdots \int \frac{1}{(2 \pi)^{k / 2}} \exp \left[-12 \sum_{i=1}^{k} s_{i}^{2}\right] \cdot \int \cdots \int \frac{1}{(2 \pi)^{k / 2}} \exp \left[-\frac{1}{2} \sum_{i=1}^{k} r_{i}^{2}\right] \prod_{l=1}^{k} d r_{i} \prod_{i=1}^{k} d s_{i} \\
& \left(\boldsymbol{s}+\boldsymbol{L}^{-1} \boldsymbol{a}\right)^{\prime}\left(\boldsymbol{s}+\boldsymbol{L}^{-1} \boldsymbol{a}\right)<\chi_{k^{2}}(\alpha) \quad \boldsymbol{r}_{k}<\boldsymbol{n}_{k} / l_{k k} \\
& r_{k-1}<\left(n_{k-1}-l_{k-1, k} r_{k}\right) / l_{k-1, k-1} \\
& r_{1}<\left(n_{1}-\sum_{j=2}^{k} l_{1 j} r_{j}\right) / l_{11}
\end{aligned}
$$

where $m_{i} \equiv \frac{N_{1}+N_{2}}{\sqrt{\sigma_{i i}}} u_{i}-\frac{N_{1} \mu_{i}^{(1)}+N_{2} \mu_{i}^{(2)}}{N_{1}+N_{2}}(i=1,2, \cdots, k), n_{i} \equiv \frac{V \overline{N_{1}+N_{2}}}{\sqrt{\sigma_{i i}}}\left(u_{i}-\mu_{i}^{(1)}\right)$ $+\sqrt{N_{2}} \sum_{j=i}^{k} l_{i j} s_{j}$, and lij's are elements of a matrix $L$ such that 


$$
\begin{aligned}
& \boldsymbol{L}==\begin{array}{lllll}
l_{11} & l_{12} & l_{13} & \cdots & l_{1 k}
\end{array}, l_{i i}>0 \quad(i=1,2, \cdots, k), \\
& \begin{array}{lllll}
0 & l_{22} & l_{23} & \cdots & l_{2 k}
\end{array} \\
& \begin{array}{lllll}
0 & 0 & l_{33} & \cdots & l_{3 k}
\end{array} \\
& \begin{array}{lllll}
0 & 0 & 0 & \cdots & l_{k k}
\end{array}
\end{aligned}
$$

\section{$\boldsymbol{L}^{\prime} \boldsymbol{R}^{-1} \boldsymbol{L}=$ unit matrix}

where

$$
\boldsymbol{R}=\left(\begin{array}{cccc}
1 & \rho_{12} & \cdots & \rho_{1 k} \\
\rho_{12} & 1 & \cdots & \rho_{2 k} \\
\vdots & \vdots & \vdots & \vdots \\
\rho_{1 k} & \rho_{2 k} & \cdots & 1
\end{array}\right)
$$

Proof. Now let us put for a moment

$$
\begin{array}{r}
\boldsymbol{y}^{(i) \prime}=\left(y_{1}^{(i)}, y_{2}^{(i)}, \cdots, y_{k}^{(i)}\right)=\left(\frac{\bar{x}_{i=}^{(i)}-\mu_{1}^{(i)}}{\sqrt{\sigma_{11} / V} N_{i}}, \frac{\bar{x}_{2}^{(i)}-\mu_{2}^{(i)}}{\sqrt{\sigma_{22}} / \sqrt{ } / N_{i}}, \cdots, \frac{\overline{\boldsymbol{x}}_{k}^{(i)}-\mu_{k}^{(i)}}{\sqrt{\sigma_{k k} / V N_{i}}}\right) \\
(i=1,2),
\end{array}
$$

then the joint elementary probability of $\left(y_{1}^{(1)}, y_{2}^{(1)}, \cdots, y_{k}^{(1)}, y_{1}^{(2)}, y_{2}^{(2)}, \cdots, y_{k}^{(2)}\right)$ may be given by

$$
\frac{1}{(2 \pi)^{k} \mid \boldsymbol{R}} \exp \left[-\frac{1}{2} \sum_{i=1}^{2} \boldsymbol{y}^{(i) \prime} \boldsymbol{R}^{-1} \boldsymbol{y}^{(i)}\right] \prod_{i=1}^{2} \prod_{j=1}^{k} d y_{j}^{(i)} .
$$

Further, the following orthogonal transformation

$$
p=\sqrt{\frac{N_{1} N_{2}}{N_{1}+N_{2}}}\left(-\frac{\boldsymbol{y}^{(1)}}{\sqrt{N_{1}}}+\frac{\boldsymbol{y}^{(2)}}{\sqrt{N_{2}}}\right), \quad \boldsymbol{q}=\sqrt{\frac{N_{1} N_{2}}{N_{1}+N_{2}}}\left(\frac{\boldsymbol{y}^{(1)}}{V / N_{2}}+\frac{\boldsymbol{y}^{(2)}}{V N_{1}}\right)
$$

gives us the elementary probability of $p_{1}, p_{2}, \cdots, p_{k}, q_{1}, q_{2}, \cdots, q_{k}$

$$
h\left(\boldsymbol{p}^{\prime}, \boldsymbol{q}^{\prime}\right) \prod_{i=1}^{k}\left(d p_{i} d q_{i}\right)=\frac{1}{(2 \pi)^{k}|\boldsymbol{R}|} \exp \left[-\frac{1}{2}\left(\boldsymbol{p}^{\prime} \boldsymbol{R}^{-1} \boldsymbol{p}+\boldsymbol{q}^{\prime} \boldsymbol{R}^{-1} \boldsymbol{q}\right)\right] \prod_{i=1}^{k}\left(d p_{i} d q_{i}\right)
$$

Now let the sample space be devided into two distinct sets $D_{1}$ and $D_{2}$ which are defined as a domain $\boldsymbol{U}^{\prime} \boldsymbol{U}=(\boldsymbol{p}+\boldsymbol{a})^{\prime} \boldsymbol{R}^{-1}(\boldsymbol{p}+\boldsymbol{a}) \leq \chi_{k}^{2}(\alpha)$, where $\quad \boldsymbol{a}=\sqrt{\frac{N_{1} N_{2}}{N_{1}+N_{2}}} c^{-1}\left(\boldsymbol{\mu}^{(2)}-\boldsymbol{\mu}^{(1)}\right)$

$$
\boldsymbol{c}=\left(\begin{array}{cccc}
\sqrt{\sigma_{11}} & 0 & \cdots & 0 \\
0 & \sqrt{\sigma_{22}} & \cdots & 0 \\
\vdots & \vdots & & \vdots \\
0 & 0 & \cdots & \sqrt{\sigma_{k k}}
\end{array}\right),
$$

and the complement respectively. Then we may and shall decompose the 
probability (1.1) into two parts.

$$
\operatorname{Pr} .\{\boldsymbol{x}<\boldsymbol{u}\}=\operatorname{Pr}\left\{\frac{N_{1} \boldsymbol{x}^{(1)}+N_{2} \boldsymbol{x}^{(2)}}{N_{1}+N_{2}}<\boldsymbol{u}, D_{1}\right\}+\operatorname{Pr} .\left\{\boldsymbol{x}^{(1)}<\boldsymbol{u}, D_{2}\right\},
$$

where $\operatorname{Pr} .\left\{\overline{\boldsymbol{x}}<\boldsymbol{u}, D_{1}\right\}$ means the probability that two events happen simultaneously and similar for other cases.

On the other hand in view of (1.2) and (1.4), we may obtain that

$$
\begin{aligned}
& \frac{N_{1} \overline{\boldsymbol{x}}^{(1)}+N_{2} \overline{\boldsymbol{x}}^{(2)}}{N_{1}+N_{2}}=\boldsymbol{E}+\boldsymbol{F} \boldsymbol{q}=\boldsymbol{\mu}^{(1)}+\boldsymbol{F} \boldsymbol{q}+\frac{N_{2}}{N_{1}+N_{2}}\left(\boldsymbol{\mu}^{(2)}-\boldsymbol{\mu}^{(1)}\right) \\
& \overline{\boldsymbol{x}}=\boldsymbol{\mu}^{(1)}+\boldsymbol{F} \boldsymbol{q}-G \boldsymbol{F} \boldsymbol{p},
\end{aligned}
$$

where

$$
\boldsymbol{E}=\frac{N_{1} \boldsymbol{\mu}^{(1)}+N_{2} \boldsymbol{\mu}^{(2)}}{N_{1}+N_{2}}, \quad \boldsymbol{F}=\frac{1}{\sqrt{N_{1}+N_{2}}}\left(\begin{array}{cccc}
\sqrt{\sigma_{11}} & 0 & \cdots & 0 \\
0 & \sqrt{\sigma_{22}} & \cdots & 0 \\
\vdots & \vdots & \ddots & \vdots \\
0 & 0 & \cdots & \sigma_{k k}
\end{array}\right), \text { and } G=\sqrt{\prime} \bar{N}_{2}
$$

Then the first term of (1.6) may be given by

$$
\operatorname{Pr} \cdot\left\{\frac{N_{1} \ddot{\boldsymbol{x}}^{(1)}+N_{2} \overline{\boldsymbol{x}}^{(2)}}{N_{1}+N_{2}}<\boldsymbol{u}, D_{1}\right\}=\int_{\substack{\boldsymbol{q}<\boldsymbol{F}^{-1}(\boldsymbol{u}-\boldsymbol{E}) \\(\boldsymbol{p}+\boldsymbol{a})^{\prime} \boldsymbol{R}^{-1}(\boldsymbol{p}+\boldsymbol{a}) \leq \chi_{k}^{2}(\alpha)}} \ldots \int_{i=1}^{k} h\left(\boldsymbol{p}^{\prime}, \boldsymbol{q}^{\prime}\right) \prod_{i=1}^{k}\left(d p_{i} d q_{i}\right) .
$$

Now let $F_{\alpha \beta}(t)$ be a matrix whose diagonal elements are all one, $(\alpha$, $\beta$ ) element $(\alpha \neq \beta)$ is $t$, and another elements are all zero. Then

$$
\begin{aligned}
& \boldsymbol{R}_{1}=\boldsymbol{R}^{-1} E_{12}\left(-\frac{\rho^{12}}{\rho^{11}}\right) \boldsymbol{E}_{13}\left(-\frac{\rho^{13}}{\rho^{11}}\right) \cdots \boldsymbol{E}_{1 k}\left(-\frac{\rho^{1 k}}{\rho^{11}}\right) \\
& =\left(\begin{array}{cccc}
\rho^{11} & \rho^{12} & \cdots & \rho^{1 k} \\
\rho^{21} & \rho^{22} & \cdots & \rho^{2 k} \\
\vdots & \vdots & & \vdots \\
\vdots & \vdots & . & \vdots \\
\rho^{k 1} & \rho^{k 2} & \cdots & \rho^{k k}
\end{array}\right)\left(\begin{array}{cccccc}
1 & -\frac{\rho^{12}}{\rho^{11}} & 0 & \cdots & 0 \\
0 & 1 & 0 & \cdots & 0 \\
0 & 0 & 1 & \cdots & 0 \\
\vdots & \vdots & \vdots & \cdot & \vdots \\
\vdots & \vdots & \vdots & \ddots & \vdots \\
0 & 0 & 0 & \cdots & 1
\end{array}\right) \quad\left(\begin{array}{ccccc}
1 & 0 & \cdots & -\frac{\rho^{1 k}}{\rho_{11}} \\
0 & 1 & \cdots & 0 \\
\vdots & \vdots & & & \vdots \\
\vdots & \vdots & & & \vdots \\
0 & 0 & \cdots & 1
\end{array}\right)
\end{aligned}
$$

becomes a matrix whose elements of first row are all zero except (1.1) element. Similarly, all elements of the second row of the matrix

$$
\boldsymbol{R}_{2}=\boldsymbol{R}_{1} E_{23}\left(-\frac{\rho_{1}^{23}}{\rho_{1}^{22}}\right) E_{24}\left(-\frac{\rho_{1}^{24}}{\rho^{22}}\right) \cdots \cdots E_{2 k}\left(-\frac{\rho_{1}^{2 k}}{\rho_{1}^{22}}\right)
$$




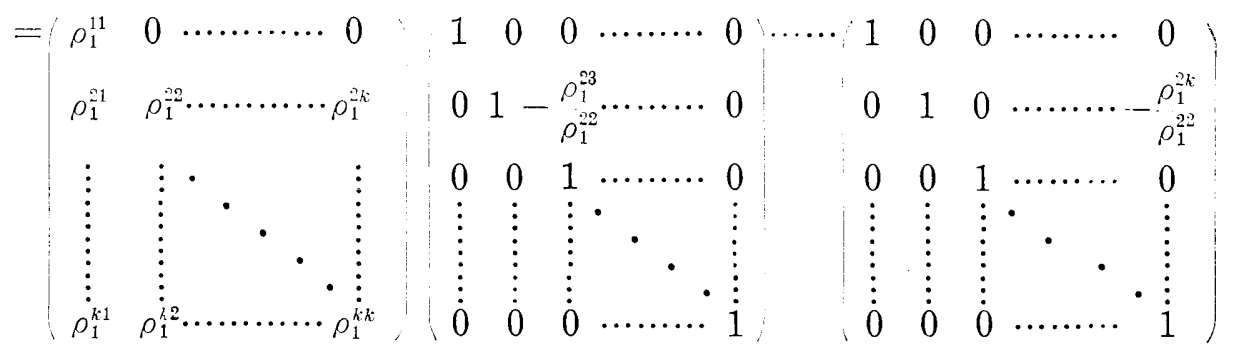

are zero except (2.1) and (2.2) elements, and so on. Thus

$$
\begin{gathered}
\boldsymbol{R}_{k-1}=\boldsymbol{R}^{-1} E_{12}\left(-\frac{\rho^{12}}{\rho^{11}}\right) \cdots \cdots E_{1 k}\left(-\frac{\rho^{1 k}}{\rho^{11}}\right) \cdot E_{23}\left(-\frac{\rho_{1}^{23}}{\rho_{1}^{22}}\right) \cdots \cdots E_{2 k}\left(-\frac{\rho_{1}^{2 k}}{\rho_{1}^{\frac{2 k}{2}}}\right) \\
\cdots \cdots E_{k-1 . k}\left(-\frac{\rho_{k-2,2}^{k-1, k}}{\rho_{k-2}^{k-1, k-1}}\right)=\boldsymbol{R}^{-1} E_{*}, \text { and } E_{*}
\end{gathered}
$$

are matrices of the following form respectively,

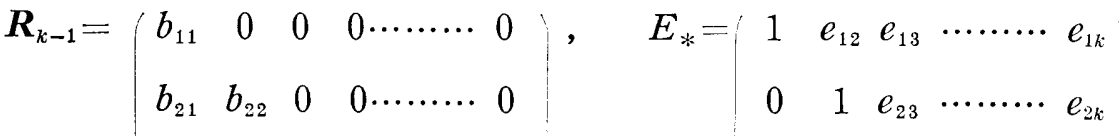

$$
\begin{aligned}
& \begin{array}{llllll}
b_{31} & b_{32} & b_{33} & 0 & \cdots \cdots \cdots & 0
\end{array} \\
& \begin{array}{lllll}
b_{k 1} & b_{k 2} & b_{k 3} & b_{k 4} & \cdots \cdots \cdots \\
b_{k i}
\end{array}
\end{aligned}
$$

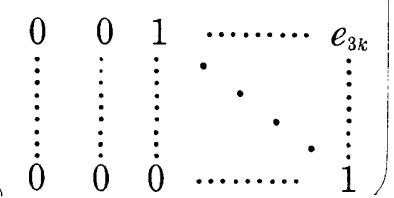

Now, if we multiply $E_{*}^{\prime}$ by $\boldsymbol{R}_{k-1}$, we get a diagonal matrix such that

$$
E_{*}^{\prime} \boldsymbol{R}_{k-1}=E_{*}^{\prime} \boldsymbol{R}^{-1} E_{*}=\left(\begin{array}{ccccc}
\lambda_{1} & 0 & \cdots \cdots \cdots \cdots & 0 \\
0 & \lambda_{2} & \cdots \cdots \cdots & 0 \\
\vdots & \vdots & \cdots & & \vdots \\
\vdots & \vdots & & & \vdots \\
\vdots & \vdots & & & \vdots \\
0 & 0 & \cdots \cdots \cdots & \lambda_{k}
\end{array}\right)
$$

and $\lambda_{i}>0(i=1,2, \cdots, k)$. Therefore, if we put

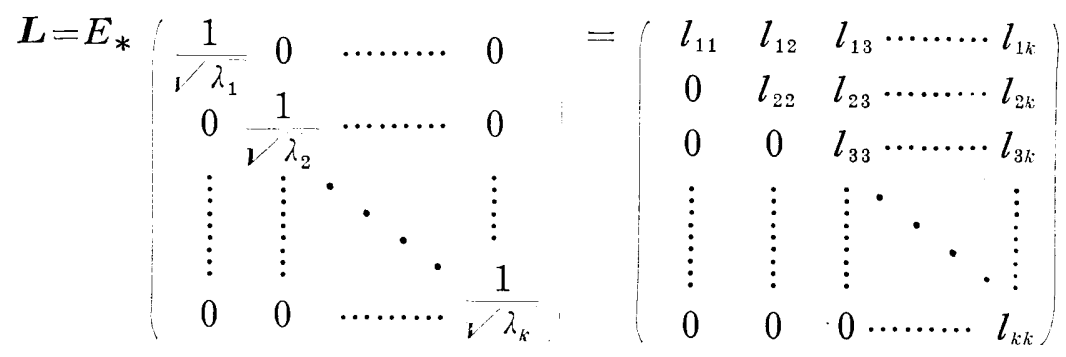

then

$$
\boldsymbol{L}^{\prime} \boldsymbol{R}^{-1} \boldsymbol{L}=\boldsymbol{I}
$$


where $\boldsymbol{I}$ is the unit matrix of $k$ degree, and $l_{i i}>0(i=1,2, \cdots, k)$. By using the following transformations

$$
\boldsymbol{p}=\boldsymbol{L} \boldsymbol{s}, \boldsymbol{q}=\boldsymbol{L} \boldsymbol{r},
$$

where $\boldsymbol{s}^{\prime}=\left(s_{1}, s_{2}, \cdots, s_{k}\right), \boldsymbol{r}=\left(\boldsymbol{r}_{1}, r_{2}, \cdots, \boldsymbol{r}_{k}\right)$, the probability (1.8) may be written in the following way:

$$
\begin{aligned}
& \operatorname{Pr} \cdot\left\{\frac{N_{1} \overline{\boldsymbol{x}}^{(1)}+N_{2} \overline{\boldsymbol{x}}^{(2)}}{N_{1}+N_{2}}<\boldsymbol{u}, D_{1}\right\}=\int_{\boldsymbol{L} \boldsymbol{r}<\boldsymbol{F}^{-1}(\boldsymbol{u}-\boldsymbol{E})} \ldots \int_{(2 \pi)^{k / 2}} \exp \left[-\frac{1}{2} \sum_{i=1}^{k} \boldsymbol{r}_{i}^{2}\right] \prod_{i=1}^{k} d r_{i} \\
& \cdot \int_{(\boldsymbol{L} \boldsymbol{s}+\boldsymbol{a})^{\prime} \boldsymbol{R}^{-1}(\boldsymbol{L} \boldsymbol{s}+\boldsymbol{a}) \leq \chi_{k}^{2}(\alpha)} \frac{1}{(2 \pi)^{k / 2}} \exp \left[-\frac{1}{2} \sum_{i=1}^{k} s_{i}^{2}\right] \prod_{i=1}^{k} d s_{i} .
\end{aligned}
$$

Let us put $\left(\boldsymbol{F}^{-1}(\boldsymbol{u}-\boldsymbol{E})\right)^{\prime}=\boldsymbol{m}^{\prime}=\left(m_{1}, \boldsymbol{m}_{2}, \cdots, m_{k}\right)$ where $m_{i}=\frac{\sqrt{N_{1}+N_{2}}}{\sqrt{\sigma_{i i}^{-}}}$

$$
\left(u_{i}-\frac{N_{1} \mu_{i}^{(1)}+N_{2} \mu_{i}^{(2)}}{\sqrt{N_{1}+N_{2}}}\right),
$$

then

$$
\begin{aligned}
& \text { (1.10) } \operatorname{Pr}\left\{\frac{N_{1} \overline{\boldsymbol{x}}^{(1)}+N_{2} \overline{\boldsymbol{x}}^{(2)}}{N_{1}+N_{2}}<\boldsymbol{u}, D_{1}\right\} \\
& =\int \cdots \int \frac{1}{(2 \pi)^{k / 2}} \exp \left[-\frac{1}{2} \sum_{i=1}^{k} r_{i}^{2}\right] \prod_{i=1}^{k} d r_{i} \int \cdots \int \frac{1}{(2 \pi)^{k / 2}} \exp \left[-\frac{1}{2}\right] \prod_{i=1}^{k} d s_{i} \\
& \boldsymbol{r}_{k}<m_{k} / l_{k k} \quad\left(\boldsymbol{s}+\boldsymbol{L}^{-1} \boldsymbol{a}\right)^{\prime}\left(\boldsymbol{s}+\boldsymbol{L}^{-1} \boldsymbol{a}\right) \leq \chi_{k^{2}}(\alpha) \\
& r_{k-1}<\left(m_{k-1}-l_{k-1, k} r_{k}\right) / l_{k-1, k-1} \\
& r_{1}<\left(m_{1}-\sum_{j=2}^{k} l_{1 j} r_{j}\right) / l_{11}
\end{aligned}
$$

The second term of (1.1) may be similarly given as follows:

$$
\begin{aligned}
& \operatorname{Pr} .\left\{\overline{\boldsymbol{x}}<\boldsymbol{u}, D_{2}\right\}=\int \cdots \int h\left(\boldsymbol{p}^{\prime}, \boldsymbol{q}^{\prime}\right) \prod_{i=1}^{k}\left(d p_{i} d q_{i}\right) \\
& \boldsymbol{q}<\boldsymbol{F}^{-1}\left(\boldsymbol{u}-\mu^{(1)}\right)+G \boldsymbol{p} \\
& (\boldsymbol{p}+\boldsymbol{a})^{\prime} \boldsymbol{R}^{-1}(\boldsymbol{p}+\boldsymbol{a})>\chi_{k^{2}}(\alpha) \\
& =\int \cdots \int \frac{1}{(2 \pi)^{k / 2}} \exp \left[-\frac{1}{2} \sum_{i=1}^{k} r_{i}^{2}\right] \cdot \frac{1}{(2 \pi)^{k / 2}} \exp \left[-\frac{1}{2} \sum_{i=1}^{k} s_{i}^{2}\right] \prod_{i=1}^{k} d r_{i} \prod_{i=1}^{k} d s_{i} \\
& \boldsymbol{L} \boldsymbol{r}<\boldsymbol{F}^{-1}\left(\boldsymbol{u}-\mu^{(1)}\right)+G \boldsymbol{L} \boldsymbol{S} \\
& (\boldsymbol{L} \boldsymbol{s}+\boldsymbol{a})^{\prime} \boldsymbol{R}^{-1}(\boldsymbol{L} \boldsymbol{s}+\boldsymbol{a})>\chi_{k^{2}}(\alpha) \\
& =\int \cdots \int \frac{1}{(2 \pi)^{k / 2}} \exp \left[-\frac{1}{2} \sum_{i=1}^{k} s_{i}^{2}\right] \cdot \int \cdots \int \frac{1}{(2 \pi)^{k / 2}} \exp \left[-\frac{1}{2} \sum_{i=1}^{k} r_{i}^{2}\right] \prod_{i=1}^{k} d r_{i} \prod_{i=1}^{k} d s_{i} \\
& \left(\boldsymbol{s}+\boldsymbol{L}^{-1} \boldsymbol{a}\right)^{\prime}\left(\boldsymbol{s}+\boldsymbol{L}^{-1} \boldsymbol{a}\right)>\chi_{k^{2}}(\alpha) \quad \boldsymbol{r}_{k}<\boldsymbol{n}_{k} / l_{k k} \\
& r_{k-1}<\left(n_{k-1}-l_{k-1, k} r_{k}\right) / l_{k-1, k-1} \\
& r_{1}<\left(n_{1}-\sum^{\left.k_{j}=2 l_{i}, r_{j}\right) / l_{11}}\right.
\end{aligned}
$$


where $\quad \boldsymbol{n}^{\prime}=\left(\boldsymbol{n}_{1}, n_{2}, \cdots, \boldsymbol{n}_{k}\right) \equiv\left(\boldsymbol{F}^{-1}\left(\boldsymbol{u}-\boldsymbol{\mu}^{(1)}\right)+G \boldsymbol{L} \boldsymbol{s}\right)^{\prime}$.

Thus we obtain the second term of the right-hand side of (1.1). In combination of (1.10) and (1.11), we may obtain (1.1) to be proved.

Theorem 1.2. The mean vector $E\{\overline{\boldsymbol{x}}\}$ and the mean square error $M$. S.E. $\{\overline{\boldsymbol{x}}\}$ of the estimate $\overline{\boldsymbol{x}}$ are given by

$$
E\{\overline{\boldsymbol{x}}\}=\boldsymbol{\mu}^{(1)}+\frac{N_{2}}{N_{1}+N_{2}}\left(\boldsymbol{\mu}^{(2)}-\boldsymbol{\mu}^{(1)}\right) \operatorname{Pr} .\left\{D_{1}\right\}-/ \frac{N_{2}}{\left(N_{1}+N_{2}\right) N_{1}} \boldsymbol{c} I_{D_{2}}\{\boldsymbol{p}\}
$$

and

$$
\begin{aligned}
& \text { M.S,E. }\{\overline{\bar{x}}\}=\frac{1}{N_{1}+N_{2}} \boldsymbol{\Sigma}+\left(\frac{N_{2}}{N_{1}+N_{2}^{-}}\right)^{2}\left(\boldsymbol{\mu}^{(2)}-\boldsymbol{\mu}^{(1)}\right)\left(\boldsymbol{\mu}^{(2)}-\boldsymbol{\mu}^{(1)}\right)^{\prime} \operatorname{Pr} .\left\{D_{1}\right\} \\
& +\frac{N_{2}}{\left(N_{1}+N_{2}\right) N_{1}} I_{D_{2}}\left\{\boldsymbol{c} \boldsymbol{p} \boldsymbol{p}^{\prime} \boldsymbol{c}^{\prime}\right\}
\end{aligned}
$$

where we put

$$
\begin{aligned}
\operatorname{Pr} .\left\{D_{1}\right\}= & \int \ldots \int \frac{1}{(2 \pi)^{\frac{k / 2}{2} \mid \boldsymbol{R}^{1 / 2}}} \exp \left[-\frac{1}{2} \boldsymbol{p}^{\prime} \boldsymbol{R}^{-1} \boldsymbol{p}\right] \prod_{i=1}^{k} d p_{i} \\
& (\boldsymbol{p}+\boldsymbol{a})^{\prime} \boldsymbol{R}^{-1}(\boldsymbol{p}+\boldsymbol{a}) \leq \chi_{k^{2}}(\alpha)
\end{aligned}
$$

$$
\begin{aligned}
I_{D_{2}}\{\boldsymbol{p}\}= & \int \ldots \int \boldsymbol{p} \cdot \frac{1}{(2 \pi)^{k / 2} \boldsymbol{R}_{i}^{1 / 2}} \exp \left[-\frac{1}{2} \boldsymbol{p}^{\prime} \boldsymbol{R}^{-1} \boldsymbol{p}\right] \prod_{i=1}^{k} d p_{i} \\
& (\boldsymbol{p}+\boldsymbol{a})^{\prime} \boldsymbol{R}^{-1}(\boldsymbol{p}+\boldsymbol{a})>\chi_{k}{ }^{2}(\alpha)
\end{aligned}
$$

Proof. Making use of the notation in the enunciation and proof of theorem 1.1., we may write

$$
\begin{aligned}
E\{\overline{\boldsymbol{x}}\} & =\int \cdots \int_{D_{1}} \boldsymbol{\mu}^{(1)}+\boldsymbol{F} \boldsymbol{q}+\frac{N_{2}}{N_{1}+N_{2}}\left(\boldsymbol{\mu}^{(2)}-\boldsymbol{\mu}^{(1)}\right) h\left(\boldsymbol{p}^{\prime}, \boldsymbol{q}^{\prime}\right) \prod_{i=1}^{k}\left(d p_{i} d q_{i}\right) \\
& +\int \cdots \int_{D_{2}}\left(\boldsymbol{\mu}^{(1)}+\boldsymbol{F} \boldsymbol{q}-G \boldsymbol{F} \boldsymbol{p}\right) h\left(\boldsymbol{p}^{\prime}, \boldsymbol{q}^{\prime}\right) \prod_{i=1}^{k}\left(d p_{i} d q_{i}\right) \\
& =\boldsymbol{\mu}^{(1)}+\frac{N_{2}}{N_{1}+N_{2}}\left(\boldsymbol{\mu}^{(2)}-\boldsymbol{\mu}^{(1)}\right) \operatorname{Pr} .\left\{D_{1}\right\}-\sqrt{\frac{N_{2}}{\left(N_{1}+N_{2}\right) N_{1}}} \boldsymbol{c} I_{D_{2}}\{\boldsymbol{p}\},
\end{aligned}
$$

because, $E\{\boldsymbol{q}\}=0$.

Thus the mean vector (1.12) is obtained. The mean square error matrix may be obtained from a following relation:

$$
\begin{array}{r}
E\left\{\boldsymbol{x} \overline{\boldsymbol{x}}^{\prime}\right\}=\int \ldots \int_{D_{1}}\left(\boldsymbol{\mu}^{(1)}+\boldsymbol{F} \boldsymbol{q}+\frac{N_{2}}{N_{1}+N_{2}}\left(\boldsymbol{\mu}^{(2)}-\boldsymbol{\mu}^{(1)}\right)\right)\left(\boldsymbol{\mu}^{(1)}+\boldsymbol{F} \boldsymbol{q}\right. \\
\left.+\frac{N_{2}}{N_{1}+N_{2}}\left(\boldsymbol{\mu}^{(2)}-\boldsymbol{\mu}^{(1)}\right)\right)^{\prime} h\left(\boldsymbol{p}^{\prime}, \boldsymbol{q}^{\prime}\right) \prod_{i=1}^{k}\left(d p_{i} d q_{i}\right)
\end{array}
$$




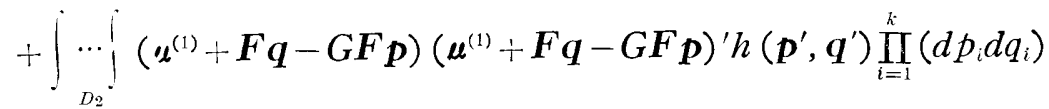

$$
\begin{aligned}
& =\int_{D_{1}} \cdots \boldsymbol{\mu}^{(1)} \boldsymbol{\mu}^{(1) \prime}+\boldsymbol{F} \boldsymbol{q} \boldsymbol{q}^{\prime} \boldsymbol{F}^{\prime}+\frac{N_{2}}{N_{1}+\bar{N}_{2}}\left(\boldsymbol{\mu}^{(1)}\left(\boldsymbol{\mu}^{(2)}-\boldsymbol{\mu}^{(1)}\right)^{\prime}+\left(\boldsymbol{\mu}^{(2)}-\boldsymbol{\mu}^{(1)}\right) \boldsymbol{\mu}^{(1) \prime}\right) \\
& +\left(\frac { N _ { 2 } } { N _ { 1 } + N _ { 2 } } \left({ }^{2}\left(\boldsymbol{\mu}^{(2)}-\boldsymbol{\mu}^{(1)}\right)\left(\boldsymbol{\mu}^{(2)}-\boldsymbol{\mu}^{(1)}\right)^{\prime}+\left(\boldsymbol{\mu}^{(1)} \boldsymbol{q}^{\prime} \boldsymbol{F}^{\prime}+\boldsymbol{F} \boldsymbol{q} \boldsymbol{\mu}^{(1)}\right)\right.\right. \\
& +\frac{N_{2}}{N_{1}+N_{2}}\left(\boldsymbol{F} \boldsymbol{q}\left(\boldsymbol{\mu}^{(2)}-\boldsymbol{\mu}^{(1)}\right)^{\prime}+\left(\boldsymbol{\mu}^{(2)}-\boldsymbol{\mu}^{(1)}\right) \boldsymbol{q}^{\prime} \boldsymbol{F}^{\prime}\right) h\left(\boldsymbol{p}^{\prime}, \boldsymbol{q}^{\prime}\right) \prod_{i=1}^{k}\left(d p_{i} d q_{i}\right) \\
& \left.+\int \underset{D_{2}}{\int} \boldsymbol{\mu}^{(1)} \boldsymbol{\mu}^{(1) \prime}+\boldsymbol{F} \boldsymbol{q} \boldsymbol{q}^{\prime} \boldsymbol{F}^{\prime}+G^{2} \boldsymbol{F} \boldsymbol{p} \boldsymbol{p}^{\prime} \boldsymbol{F}^{\prime}+\left(\boldsymbol{\mu}^{(1)} \boldsymbol{q}^{\prime} \boldsymbol{F}^{\prime}+\boldsymbol{F} \boldsymbol{q} \boldsymbol{\mu}^{(1) \prime}\right)-G_{\left(\boldsymbol{\mu}^{(1)}\right.} \boldsymbol{p}^{\prime} \boldsymbol{F}^{\prime}+\boldsymbol{F} \boldsymbol{p} \boldsymbol{\mu}^{(1) \prime}\right) \\
& -G\left(\boldsymbol{F} \boldsymbol{q} \boldsymbol{p}^{\prime} \boldsymbol{F}^{\prime}+\boldsymbol{F} \boldsymbol{p} \boldsymbol{q}^{\prime} \boldsymbol{F}^{\prime}\right) h\left(\boldsymbol{p}^{\prime}, \boldsymbol{q}^{\prime}\right) \prod_{z=1}^{k}\left(d p_{i} d q_{i}\right) .
\end{aligned}
$$

Then substituting the following relations

$$
\begin{aligned}
& I_{D_{1}+D_{2}}\left\{\boldsymbol{q} \boldsymbol{q}^{\prime}\right\}=\left.\int_{D_{1}+D_{2}} \ldots\right|_{\boldsymbol{q}} \boldsymbol{q} \boldsymbol{q}^{\prime} h\left(\boldsymbol{p}^{\prime}, \boldsymbol{q}^{\prime}\right) \prod_{i=1}^{k}\left(d p_{i} d q_{i}\right)=\boldsymbol{R}, \\
& \boldsymbol{I}_{D_{1}+D_{2}}\{\boldsymbol{q}\}=\boldsymbol{I}_{D_{1}}\{\boldsymbol{q}\}=0, \quad I_{D_{2}}\left\{\boldsymbol{q} \boldsymbol{p}^{\prime}\right\}=\boldsymbol{I}_{D_{2}}\left\{\boldsymbol{p} \boldsymbol{q}^{\prime}\right\}=0
\end{aligned}
$$

for (1.17), we may obtain that

$$
\begin{aligned}
& E\left\{\boldsymbol{x} \boldsymbol{x}^{\prime}\right\}=\boldsymbol{\mu}^{(1)} \boldsymbol{\mu}^{(1) \prime}+\frac{1}{N_{1}+N_{2}} \boldsymbol{\Sigma}+\frac{N_{2}}{N_{1}+N_{2}}\left\{\boldsymbol{\mu}^{(1)}\left(\boldsymbol{\mu}^{(2)}-\boldsymbol{\mu}^{(1)}\right)^{\prime}+\left(\boldsymbol{\mu}^{(2)}-\boldsymbol{\mu}^{(1)}\right) \boldsymbol{\mu}^{(1) \prime}\right\} \operatorname{Pr} \cdot\left\{D_{1}\right\} \\
& +\left(\frac{N_{2}}{N_{1}+N_{2}}\right)^{2}\left(\boldsymbol{\mu}^{(2)}-\boldsymbol{\mu}^{(1)}\right)\left(\boldsymbol{\mu}^{(2)}-\boldsymbol{\mu}^{(1)}\right)^{\prime} \operatorname{Pr}\left\{\left\{D_{1}\right\}+\frac{N_{2}}{N_{1}} I_{D_{2}}\left\{\boldsymbol{F} \boldsymbol{p} \boldsymbol{p}^{\prime} \boldsymbol{F}^{\prime}\right\}\right. \\
& \quad-\sqrt{\frac{N_{2}}{N_{1}}} I_{D_{2}}\left\{\boldsymbol{\mu}^{(1)} \boldsymbol{p}^{\prime} \boldsymbol{F}^{\prime}+\boldsymbol{F} \boldsymbol{p} \boldsymbol{\mu}^{(1) \prime}\right\} .
\end{aligned}
$$

Thus the mean square error matrix M.S.E. $\{\boldsymbol{x}\}$ of the estimate $\overline{\boldsymbol{x}}$ may be obtained in the following manner.

$$
\begin{aligned}
& \text { M.S.E. }\{\boldsymbol{x}\}=E\left\{\left(\overline{\boldsymbol{x}}-\boldsymbol{\mu}^{(1)}\right)\left(\overline{\boldsymbol{x}}-\boldsymbol{\mu}^{(1)}\right)^{\prime}\right\} \\
& =E\left\{\bar{x} \boldsymbol{x}^{\prime}\right\}-\boldsymbol{\mu}^{(1)} E\left\{\boldsymbol{x}^{\prime}\right\}-E\{\bar{x}\} \boldsymbol{\mu}^{(1) \prime}+\boldsymbol{\mu}^{(1)} \boldsymbol{\mu}^{(1) \prime} \\
& =\frac{1}{N_{1}+N_{2}} \sum+\left(\frac{N_{2}}{N_{1}+N_{2}}\right)^{2}\left(\mu^{(2)}-\mu^{(1)}\right)\left(\mu^{(2)}-\mu^{(1)}\right)^{\prime} P r \cdot\left\{D_{1}\right\}
\end{aligned}
$$

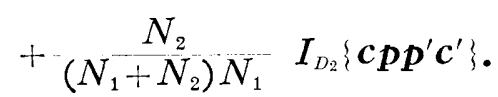

Corollary 1.1. Specially when $\boldsymbol{\mu}^{(2)}=\boldsymbol{\mu}^{(1)}, E\{\overline{\boldsymbol{x}}\}=\boldsymbol{\mu}^{(1)}$, that is, $\ddot{x}$ is an unbiased estimate of $\mu^{(1)}$, but the variance components and the absolute value of covariance components of the mean square error matrix are greater 
than $\sigma_{i i} /\left(N_{1}+N_{2}\right)$ and $\sigma_{i j} /\left(N_{1}+N_{2}\right)$ and less than $\sigma_{i i} / N_{1}$ and $\mid \sigma_{i j} / N_{1}$ respectively, $(i, j=1,2)$.

Corollary 1.2. Specially when $N_{1}$ is very large compared with $N_{2}$, that is $N_{1} \gg N_{2}$, the bias of the estimate $\bar{x}$ becomes small, and the mean square error matrix becomes $\Sigma /\left(N_{1}+N_{2}\right)$ nearly.

Corollary 1.3. Specially when $\mu^{(2)}=\mu^{(1)}$, the variance component of the M.S.E. $\{\overline{\boldsymbol{x}}\}$ in case of multivariate pooling of data becomes smaller than the variance of the corresponding estimate in case of univariate pooling of data. The difference is given by

$$
\frac{N_{2} \sigma_{i i}}{\left(N_{1}+N_{2}\right) N_{1}}-\left[I_{D_{2}}\left\{p_{i}^{2}\right\}-I_{D_{2} *}\left\{p_{i}^{2}\right\}\right]<0 \quad(i=1,2, \cdots, k)
$$

where

$$
\begin{aligned}
& I_{D_{2}}\left\{p_{i}^{2}\right\}=\int \cdots \int p_{i}^{2} \frac{1}{(2 \pi)^{k / 2}} \boldsymbol{R}^{1 / 2} \exp \left[-\frac{1}{2} \boldsymbol{p}^{\prime} \boldsymbol{R}^{-1} \boldsymbol{p}\right] \prod_{i=1}^{k} d p_{i} \\
& (\boldsymbol{p}+\boldsymbol{a})^{\prime} \boldsymbol{R}^{-1}(\boldsymbol{p}+\boldsymbol{a})>\chi_{k}{ }^{2}(\alpha) \\
& I_{D^{2} *}\left\{p_{i}^{2}\right\}=\int p_{i}^{2} \frac{1}{(2 \pi)^{1 / 2}} \exp \left[-\frac{1}{2} p_{i}^{2}\right] d p_{i} \\
& p_{i}+a_{i}>\left(\chi_{1}^{2}(\alpha)\right)^{1 / 2}
\end{aligned}
$$

2.2. Type 2. (The case with "unknown population dispersion matrix" in Type 1)

Let $O_{N_{1}}:\left(\boldsymbol{x}_{1}^{(1)}, \boldsymbol{x}_{2}^{(1)}, \cdots, \boldsymbol{x}_{v_{1}}^{(1)}\right)$ be a random sample of $N_{1}$ vector observations from a $k$-variate non-degenerate normal population $N\left[\mu^{(1)}, \Sigma\right]$ and let $O_{N_{2}}$ : $\left(\boldsymbol{x}_{1}^{(2)}, \boldsymbol{x}_{2}^{(2)}, \cdots, \boldsymbol{x}_{N_{2}}^{(2)}\right)$ be another random sample of $N_{2}$ from some $k$-variate normal populaon $N\left[\boldsymbol{\mu}^{(2)}, \Sigma\right]$. In this section, the values of these two common population dispersion matrices are assumed to be unknown to us, but the populations have not necessarily the same population mean vector. Our attempt of the present inference procedure is the same in Type 1.

Now our rule of inference procedure is as follows:

(i) Let $\overline{\boldsymbol{x}}^{(i)}$ be sample mean vector defined by $O_{N i}, i=1,2$. and let $S$ be the unbiased estimate of common population dispersion given by

$$
\boldsymbol{S}=\left(N_{1}+N_{2}-2\right)^{-1}\left\{\sum_{i=1}^{N_{1}}\left(\boldsymbol{x}_{i}^{(1)}-\overline{\boldsymbol{x}}^{(1)}\right)\left(\boldsymbol{x}_{i}^{(1)}-\boldsymbol{x}^{(1)}\right)^{\prime}+\sum_{j=1}^{N_{2}}\left(\boldsymbol{x}_{j}^{(2)}-\overline{\boldsymbol{x}}^{(2)}\right)\left(\boldsymbol{x}_{j}^{(2)}-\overline{\boldsymbol{x}}^{(2)}\right)^{\prime}\right\}
$$

(ii) Let the statistic $T^{2}$ be defined by

$$
T^{2}=\frac{N_{1} N_{2}}{N_{1}+N_{2}^{-}}\left(\bar{x}^{(2)}-\bar{x}^{(1)}\right)^{\prime} S^{-1}\left(\overline{\boldsymbol{x}}^{(2)}-\overline{\boldsymbol{x}}^{(1)}\right)
$$

(iii) Then let us define the statistic $\boldsymbol{x}$ in the following way:

(a) $\overline{\overline{\boldsymbol{x}}}=\frac{N_{1} \overline{\boldsymbol{x}}^{(1)}+N_{2} \overline{\boldsymbol{x}}^{(2)}}{N_{1}+\bar{N}_{2}}$, if $\frac{N_{1}+N_{2}-k-1}{k} \cdot \frac{T^{2}}{N_{1}+N_{2}-2} \leq F_{k, N_{1}+N_{2}-k-1}(\alpha)$ 
(b) $\cdot \overline{\boldsymbol{x}}=\overline{\boldsymbol{x}}^{(1)} \quad$, if $\frac{N_{1}+N_{2}-k-1}{k} \cdot \frac{T^{2}}{N_{1}+N_{2}-2}>F_{k, N_{1}+N_{2}-k-1}(\alpha)$ where $F_{k, N_{1}+v_{2}-k-1}(\alpha)$ denotes $\alpha$-percent point of $F$-distribution with the pair of degrees of freedom $\left(k, N_{1}+N_{2}-k-1\right)$.

Theorem 2.1. The distribution function of $x$ is given by

$$
\begin{aligned}
& \operatorname{Pr} .\{\overline{\boldsymbol{x}}<\boldsymbol{u}\} \\
& =\int \cdots \int \frac{1}{(2 \pi)^{k / 2}} \exp \left[-\frac{1}{2} \sum_{i=1}^{k} r_{i}^{2}\right] \prod_{i=1}^{k} d r \\
& r_{k}<m_{k} / l_{k k} \\
& r_{k-1}<\left(m_{k-1}-l_{k-1, k} r_{k}\right) / l_{k-1, k-1} \\
& r_{1}<\left(m_{1}-k \sum_{j=2} l_{1 j} r_{j}\right) / l_{11} \\
& \text { · } \int \cdots \cdot \frac{1}{(2 \pi)^{k / 2}} \exp \left[-\frac{1}{2} \sum_{i=1}^{k} s_{i}^{2}\right] \frac{\left(\chi^{2} / 2\right)^{\left(N_{1}+N_{2}-k-1\right) / 2-1} \exp \left[-\chi^{2} / 2\right]}{2 \Gamma\left\{\left(N_{1}+N_{2}-k-1\right) / 2\right\}}\left(\prod_{i=1}^{k} d s_{i}\right) d \chi^{2} \\
& \frac{\left(\boldsymbol{s}+\boldsymbol{L}^{-1} \boldsymbol{a}\right)^{\prime}\left(\boldsymbol{s}+\boldsymbol{L}^{-1} \boldsymbol{a}\right)}{\chi^{2}} \leq \frac{k}{N_{1}+N_{2}-k-1} F_{k, N_{1}+N_{2}-k-1}(\alpha) \\
& +\int \cdots \int \frac{1}{(2 \pi)^{\bar{k} / 2}} \exp \left[-\frac{1}{2} \sum_{i=1}^{k} s_{i}^{2}\right] \frac{\left(\chi^{2} / 2\right)^{\left(N_{1}+N_{2}-k-1\right) / 2-1} \exp \left[-\chi^{2} / 2\right]}{2 \Gamma\left\{\left(N_{1}+N_{2}-k-1\right) / 2\right\}} \\
& \frac{\left(\boldsymbol{s}+\boldsymbol{L}^{-1} \boldsymbol{a}\right)^{\prime}\left(\boldsymbol{s}+\boldsymbol{L}^{-1} \boldsymbol{a}\right)}{\chi^{2}}>\frac{k}{N_{1}+N_{2}-k-1} F_{k, N_{1}+N_{2}-k-1}(\alpha) \\
& \cdot \int \cdots \int \frac{1}{(2 \pi)^{k / 2}} \exp \left[-\frac{1}{2} \sum_{i=1}^{k} r_{i}^{2}\right]\left(\prod_{i=1}^{k} d r_{i}\right)\left(\prod_{i=1}^{k} d s_{i}\right) d \chi^{2} \\
& r_{k}<n_{k} / l_{k k} \\
& r_{k-1}<\left(n_{k-1}-l_{k-1, k} r_{k}\right) / l_{k-1, k-1} \\
& r_{1}<\left(n_{1}-k \sum{ }_{i=2} l_{1 j} r_{j}\right) / l_{11}
\end{aligned}
$$

Proof. Let us use of the notation in the enunciation of this section and proof of Theorem 1.1.

Let $\boldsymbol{D}$ be a nonsingular matrix such that $\boldsymbol{D} \Sigma \boldsymbol{D}^{\prime}=\boldsymbol{I}$ and define

$$
\boldsymbol{Y}=\sqrt{\frac{N_{1} N_{2}}{N_{1}+N_{2}}}\left(\overline{\boldsymbol{x}}^{(2)}-\overline{\boldsymbol{x}}^{(1)}\right), \mathbf{Y}^{*}=\left(\begin{array}{c}
Y_{1}^{*} \\
Y_{2}^{*} \\
\vdots \\
Y_{k}^{*}
\end{array}\right)=D Y, S^{*}=D S D^{\prime} .
$$

Then $\boldsymbol{Y}$ is distributed according to $N\left[\sqrt{\frac{N_{1} N_{2}}{N_{1}+N_{2}}}\left(\boldsymbol{\mu}^{(2)}-\boldsymbol{\mu}^{(1)}\right), \boldsymbol{\Sigma}\right]$, and $\left(N_{1}+\right.$ $\left.N_{2}-2\right) S$ is distributed as $\sum_{\beta=1}^{N_{1}+N_{2}-2} \boldsymbol{Z}_{\beta} \boldsymbol{Z}_{\beta}^{\prime}$ with the $\boldsymbol{Z}_{\beta}$ independent, each with distribution $N[\boldsymbol{O}, \boldsymbol{\Sigma}]$, and $\boldsymbol{Z}_{\beta}$ is independent of $\boldsymbol{x}^{(1)}, \ddot{\boldsymbol{x}}^{(2)}$, thereby is independent of $\boldsymbol{p}$ and $\boldsymbol{q} \cdot \boldsymbol{Y}^{*}$ is distributed according to $N \sqrt{\frac{N_{1} N_{2}}{N_{1}+N_{2}}} \boldsymbol{D}\left(\boldsymbol{\mu}^{(2)}-\boldsymbol{\mu}^{(1)}\right)$, $\boldsymbol{\Sigma}]$, and $\left(N_{1}+N_{2}-2\right) S^{*}$ is distributed as $\sum_{\beta=1}^{N_{1}+N_{2}-2} \boldsymbol{Z}_{\alpha}^{*} \boldsymbol{Z}_{\beta}^{* \prime}=\sum_{\beta=1}^{N_{1}+N_{2}-2} \boldsymbol{D} \boldsymbol{Z}_{\beta}\left(\boldsymbol{D} \boldsymbol{Z}_{\beta}\right)^{\prime}$ with 
the $\boldsymbol{Z}_{\beta}^{*}$ independent, each with distribution $N[O, I]$, and $\boldsymbol{Z}_{\beta}^{*}$ is independent of $\boldsymbol{Y}^{*}, \boldsymbol{p}$ and $\boldsymbol{q}$.

Let the first row of a $k \times k$ orthogonal matrix $Q$ be defined by

$$
\boldsymbol{q}_{1 i}=\frac{\boldsymbol{Y}_{i}^{*}}{\sqrt{\boldsymbol{Y}^{* \prime} \boldsymbol{Y}^{*}}} \quad i=1,2, \cdots, k
$$

this is permissible since $\sum_{i=1}^{k} q_{1 i}^{2}=1$. Since $Q$ depends on $\boldsymbol{Y}^{*}$ it is a random matrix. Now let

$$
\boldsymbol{V}=\boldsymbol{Q} \boldsymbol{Y}^{*}, \quad \boldsymbol{B}=\boldsymbol{Q}\left(N_{1}+N_{2}-2\right) \boldsymbol{S}^{*} \boldsymbol{Q}^{\prime}
$$

Then the conditional distribution of $\boldsymbol{B}$ given $Q$ is that of $\sum_{\beta=1}^{N_{1}+N_{2}-2} \boldsymbol{W}_{\beta} \boldsymbol{W}_{\beta}^{\prime}$ where conditionally the $\boldsymbol{W}_{\beta}$ defined by $\boldsymbol{Q} \boldsymbol{Z}_{\beta}^{*}$ are independent, each with distribution $N[O, I]$.

If we put

$$
\boldsymbol{B}=\left(b_{i j}\right)=\left(\begin{array}{cccc}
b_{11} & b_{12} & \cdots & b_{1 k} \\
b_{21} & b_{22} & \cdots & b_{2 k} \\
\vdots & \vdots & \cdots & \vdots \\
\vdots & \vdots & \cdot & \vdots \\
b_{k 1} & b_{k 2} & \cdots & b_{k k}
\end{array}\right)=\left(\begin{array}{ll}
b_{11} & b_{(1)} \\
b_{(1)}^{\prime} & \boldsymbol{B}_{22}
\end{array}\right)
$$

then $b_{11 \cdot 2 \ldots k}=b_{11}-b_{(1)} B_{22}^{-1} b_{(1)}^{\prime}$ is conditionally distributed as $\sum_{\beta=1}^{N_{1}+N_{2}-2} \bar{X}_{\beta}^{(k-1)}$ where conditionally the $X_{\beta}$ are independent, each with the distribution $N(0,1)$; that is, $b_{11 \cdot 2 \cdots k}$ is conditionally distributed as $\chi^{2}$ with $N_{1}+N_{2}-k-1$ degrees of freedom. Since the conditional distribution of $b_{112 \ldots k}$ does not depend on $Q$, it is unconditionally distributed as $\chi^{2}$, and is independent of $\boldsymbol{p}, \boldsymbol{q}$.

On the other hand, if we put

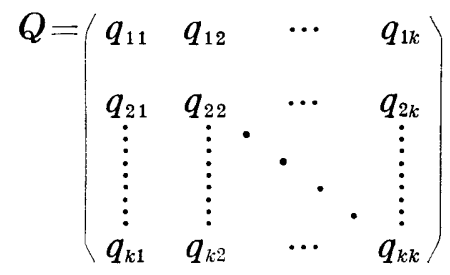

then $V_{1}=\sum_{i=1}^{k} q_{1 i} Y_{i}^{*}=\sqrt{\boldsymbol{Y}^{* \prime} \boldsymbol{Y}^{*}}, \quad V_{j}=\sum_{i=1}^{k} q_{j i} Y_{i}^{*}=V^{\prime} \overline{\boldsymbol{Y}^{* \prime} \boldsymbol{Y}^{*}} \sum_{i=1}^{k} q_{j i} q_{1 i}=0$ and

$$
\begin{aligned}
V_{1}^{2} & =\boldsymbol{Y}^{* \prime} \boldsymbol{Y}^{*}=\boldsymbol{Y}^{\prime} \boldsymbol{D}^{\prime} \boldsymbol{D} \boldsymbol{Y}=\boldsymbol{Y}^{\prime}(\boldsymbol{D} \boldsymbol{\Sigma})^{-1}(\boldsymbol{D} \boldsymbol{\Sigma}) \boldsymbol{D}^{\prime} \boldsymbol{D} \boldsymbol{Y} \\
& =\boldsymbol{Y}^{\prime} \boldsymbol{\Sigma}^{-1} \boldsymbol{D}^{-1}\left(\boldsymbol{D} \boldsymbol{\boldsymbol { \Sigma } ^ { \prime }}\right) \boldsymbol{D} \boldsymbol{Y}=\boldsymbol{Y}^{\prime} \boldsymbol{\Sigma}^{-1} \boldsymbol{Y} \\
& =\sqrt{\frac{N_{1} N_{2}}{N_{1}+N_{2}}}\left(\overline{\boldsymbol{x}}^{(2)}-\overline{\boldsymbol{x}}^{(1)}\right)^{\prime} \boldsymbol{\Sigma}^{-1} \sqrt{\frac{N_{1} N_{2}}{N_{1}+N_{2}}}\left(\overline{\boldsymbol{x}}^{(2)}-\overline{\boldsymbol{x}}^{(1)}\right) \\
& =(\boldsymbol{c}(\boldsymbol{p}+\boldsymbol{a}))^{\prime} \boldsymbol{\Sigma}^{-1}(\boldsymbol{c}(\boldsymbol{p}+\boldsymbol{a})) \\
& =(\boldsymbol{p}+\boldsymbol{a})^{\prime} \boldsymbol{c}^{\prime} \boldsymbol{\Sigma}^{-1} \boldsymbol{c}(\boldsymbol{p}+\boldsymbol{a})
\end{aligned}
$$




$$
\begin{aligned}
& =(\boldsymbol{p}+\boldsymbol{a})^{\prime}\left(\boldsymbol{c}^{-1} \boldsymbol{\Sigma}\left(\boldsymbol{c}^{\prime}\right)^{-1}\right)^{-1}(\boldsymbol{p}+\boldsymbol{a}) \\
& =(\boldsymbol{p}+\boldsymbol{a})^{\prime} \boldsymbol{R}^{-1}(\boldsymbol{p}+\boldsymbol{a})
\end{aligned}
$$

has a noncentral $\chi^{2}$-distribution with $k$ degrees of freedom. Then $T^{2} /\left(N_{1}\right.$ $\left.+N_{2}-2\right)=\boldsymbol{Y}^{\prime} \boldsymbol{S}^{-1} \boldsymbol{Y} /\left(N_{1}+N_{2}-2\right)=\boldsymbol{Y}^{* \prime} \boldsymbol{S}^{*-1} \boldsymbol{Y}^{*} /\left(N_{1}+N_{2}-2\right)$

$$
\begin{aligned}
& =V^{\prime} \boldsymbol{B}^{-1} V=\left(V_{1} 0 \cdots 0\right) / b^{11} \quad b^{12} \quad \cdots \quad b^{1 k} \quad V_{1}=V_{1}^{2} b^{11}=\frac{V_{1}^{2}}{b_{11 \cdot 2}},
\end{aligned}
$$

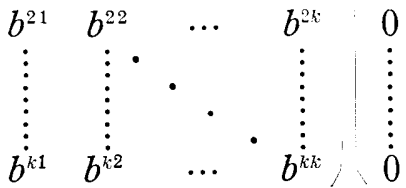

where $\left(b_{i j}\right)=\boldsymbol{B}^{-1}$, is distributed as the ratio of a noncentral $\chi^{2}$ and an independent $\chi^{2}$. Therefore if $\boldsymbol{\mu}^{(2)}=\boldsymbol{\mu}^{(1)},\left[T^{2} /\left(N_{1}+N_{2}-2\right)\right] \cdot\left[\left(N_{1}+N_{2}-k-1\right) / k\right]$ is distributed as a $F$ with $k$ and $N_{1}+N_{2}-k-1$ degrees of freedom. From above relation the inference rule of this theorem follows.

The joint probability density of $\left(\boldsymbol{p}, \boldsymbol{q}, \chi^{2}\right)$ where $\chi^{2}=b_{11 \cdot 2 \cdots k}$ may be given by

$$
\begin{gathered}
h\left(\boldsymbol{p}^{\prime}, \boldsymbol{q}^{\prime}\right) f_{N_{1}+N_{2}-k-1}\left(\chi^{2}\right)=\frac{1}{(2 \pi)^{\pi} \boldsymbol{R}} \exp \left[-\frac{1}{2}\left(\boldsymbol{p}^{\prime} \boldsymbol{R}^{-1} \boldsymbol{p}+\boldsymbol{q}^{\prime} \boldsymbol{R}^{-1} \boldsymbol{q}\right)\right] \\
\left(\chi^{2} / 2\right)^{\left(N_{1}+N_{2}-k-1\right) / 2-1} \exp \left[-\chi^{2} / 2\right] \\
2 \Gamma\left[\left(N_{1}+N_{2}-k-1\right) / 2\right]
\end{gathered}
$$

Now let the sample space be devided into two mutually distinct sets $D_{1}$ and $D_{2}$ which are defined as domains $\left[\left\{(\boldsymbol{p}+\boldsymbol{a})^{\prime} \boldsymbol{R}^{-1}(\boldsymbol{p}+\boldsymbol{a})\right\} / \chi^{2}\right]\left[\left(N_{1}+N_{2}\right.\right.$ $-k-1) / k] \leq F_{k, N^{1+N}, N_{2}-k-1}(\alpha)$ and the complement respectively. Then

$$
\operatorname{Pr} .\{\boldsymbol{x}<\boldsymbol{u}\}=\operatorname{Pr} \cdot\left\{\frac{N_{1} \boldsymbol{x}^{(1)}+N_{2} \overline{\boldsymbol{x}}^{(2)}}{N_{1}+N_{2}}<\boldsymbol{u}, D_{1}\right\}+\operatorname{Pr} \cdot\left\{\overline{\boldsymbol{x}}^{(1)}<\boldsymbol{u}, D_{2}\right\}
$$

The first term of the right-hand side of (2.2) may be

$$
\begin{aligned}
& \operatorname{Pr} \cdot\left\{\begin{array}{c}
N_{1} \overline{\boldsymbol{x}}^{(1)}+N_{2} \overline{\boldsymbol{x}}^{(2)} \\
N_{1}+N_{2}
\end{array}{ }^{2} \boldsymbol{u}, D_{1}=\int \ldots \int h\left(\boldsymbol{p}^{\prime}, \boldsymbol{q}^{\prime}\right) f_{N_{1}+N_{2}-k-1}\left(\chi^{2}\right) \prod_{i=1}^{k}\left(d p_{i} d q_{i}\right) \cdot d \chi^{2},\right. \\
& \boldsymbol{q}<\boldsymbol{F}^{-1}(\boldsymbol{u}-\boldsymbol{E}) \\
& {\left[\left\{(\boldsymbol{p}+\boldsymbol{a})^{\prime} \boldsymbol{R}^{-1}(\boldsymbol{p}+\boldsymbol{a})\right\} / \chi^{2}\right]\left[\left(N_{1}+N_{2}-k-1\right) / k\right] \leq F_{k} N_{1}+N_{2}-k-1(\alpha)}
\end{aligned}
$$

and by using the transformation (1.8), the probability may be given by

$$
\begin{aligned}
\operatorname{Pr} . & \left.\frac{N_{1} \overline{\boldsymbol{x}}^{(1)}+N_{2} \overline{\boldsymbol{x}}^{(2)}}{N_{1}+N_{2}}<\boldsymbol{u}, D_{1}\right\}=\int \ldots \int \frac{1}{(2 \pi)^{k / 2}} \exp \left[-\frac{1}{2} \boldsymbol{r}^{\prime} \boldsymbol{r}\right] \prod_{i=1}^{k} d r_{i} \\
& \cdot \int_{\boldsymbol{L} \boldsymbol{r}<\boldsymbol{F}^{-1}(\boldsymbol{u}-\boldsymbol{E})} \ldots \int_{(2 \pi)^{k / 2}} \exp \left[-\frac{1}{2} \boldsymbol{s}^{\prime} \boldsymbol{s}\right] \frac{\left(\chi^{2} / 2\right)^{\left(N_{1}+N_{2}-k-1\right) / 2-1} \exp \left[-\chi^{2} / 2\right]}{2 \Gamma\left\{\left(N_{1}+N_{2}-k-1\right) / 2\right\}} \prod_{i=1}^{k} d s_{i} d \chi^{2} \\
& {\left.\left[\{\boldsymbol{L} \boldsymbol{s}+\boldsymbol{a})^{\prime} \boldsymbol{R}^{-1}(\boldsymbol{L} \boldsymbol{s}+\boldsymbol{a})\right\} / \chi^{2}\right]\left[\left(N_{1}+N_{2}-k-1\right) / k\right] \leq F_{k, N_{1}+N_{2}-k-1}(\alpha) }
\end{aligned}
$$

Using the same notation as that of the theorem 1.1, we may obtain 
(2.3) $\operatorname{Pr} .\left\{\frac{N_{1} \boldsymbol{x}^{(1)}+N_{2} \boldsymbol{x}^{(2)}}{N_{1}+N_{2}}<\boldsymbol{u}_{1}, D_{1}\right\}$

$$
\begin{aligned}
& =\int \cdots \int \frac{1}{(2 \pi)^{k / 2}} \exp \left[-\frac{1}{2} \sum_{i=1}^{k} r_{i}^{2}\right] \prod_{i=1}^{k} d r_{i} \\
& r_{k}<m_{k} / l_{k k} \\
& r_{k-1}<\left(m_{k-1}-l_{k-1, k} \boldsymbol{r}_{k}\right) / l_{k-1, k-1} \\
& r_{1}<\left(m_{1}-k \sum j=2 l_{1 j} r_{j}\right) / l_{11} \\
& \text { - } \int \cdots \int \frac{1}{(2 \pi)^{k / 2}} \exp \left[-\frac{1}{2} \sum_{i=1}^{k} s_{i}^{2}\right] \frac{\left(\chi^{2} / 2\right)^{\left(N_{1}+N_{2}-k-1\right) / 2-1} \exp \left[-\chi^{2} / 2\right]}{\left.\left.2 \Gamma i N_{1}+N_{2}-k-1\right) / 2\right\}}\left(\prod_{i=1}^{k} d s_{i}\right) d \chi^{2} \\
& \left.\left[\left\{\boldsymbol{s}+\boldsymbol{L}^{-1} \boldsymbol{a}\right)^{\prime}\left(\boldsymbol{s}+\boldsymbol{L}^{-1} \boldsymbol{a}\right)\right\} / \chi^{2}\right] \leq\left\{k /\left(N_{1}+N_{2}-k-1\right)\right\} F_{k, N_{1}+N_{2}-k-1}(\alpha)
\end{aligned}
$$

The second term of the right-hand side of (2.2) may be similarly

$$
\begin{aligned}
\operatorname{Pr} .\left\{\overline{\boldsymbol{x}}^{(1)}<\boldsymbol{u}, D_{2}\right\} & =\int \ldots \int h\left(\boldsymbol{p}^{\prime}, \boldsymbol{q}^{\prime}\right) f_{N_{1}+N_{2}-k-1}\left(\chi^{2}\right) \prod_{i=1}^{k}\left(d p_{i} d q_{i}\right) d \chi^{2} \\
& =\boldsymbol{q}^{-1}(\boldsymbol{u}-\boldsymbol{E})+G \boldsymbol{p} \\
& {\left.\left[\left\{(\boldsymbol{p}+\boldsymbol{a})^{\prime} \boldsymbol{R}^{-1}(\boldsymbol{p}+\boldsymbol{a})\right\} / \chi^{2}\right]\left[N_{1}+N_{2}-k-1\right) / k\right]>F_{k, N_{1}+N_{2}-k-1}(\alpha) }
\end{aligned}
$$$$
\begin{aligned}
= & \int \cdots \int \frac{1}{(2 \pi)^{k / 2}} \exp \left[-\frac{1}{2}\left(\boldsymbol{r}^{\prime} \boldsymbol{r}+\boldsymbol{s}^{\prime} \boldsymbol{s}\right)\right] \frac{\left(\chi^{2} / 2\right)^{\left(N_{1}+N_{2}-k-1\right) / 2-1} \exp \left[-\chi^{2} / 2\right]}{2 \Gamma\left\{\left(N_{1}+N_{2}-k-1\right) / 2\right\}}\left(\prod_{i=1}^{k} d r_{i} d s_{i}\right) \cdot d \chi^{2} \\
& \boldsymbol{L} \boldsymbol{r}<\boldsymbol{F}^{-1}(\boldsymbol{u}-\boldsymbol{\mu}(1))+G \boldsymbol{L} \boldsymbol{s} \\
& {\left[\left\{\left(\boldsymbol{s}+\boldsymbol{L}^{-1} \boldsymbol{a}\right)^{\prime}\left(\boldsymbol{s}+\boldsymbol{L}^{-1} \boldsymbol{a}\right)\right\} / \chi^{2}\right]>\left\{k /\left(N_{1}+N_{2}-k-1\right)\right\} F_{k, N_{1}+N_{2}-k-1}(\alpha) }
\end{aligned}
$$

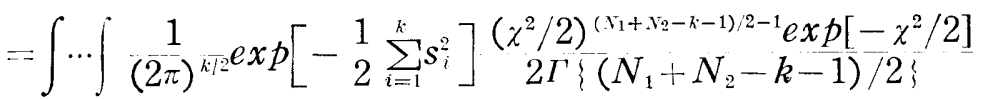$$
\left[\left\{\left(\boldsymbol{s}+\boldsymbol{L}^{-1} \boldsymbol{a}\right)^{\prime}\left(\boldsymbol{s}+\boldsymbol{L}^{-1} \boldsymbol{a}\right)\right\} / \chi^{2}\right]>\left\{k /\left(N_{1}+N_{2}-k-1\right)\right\} F_{k, N_{1}+N_{2}-k-1}(\alpha)
$$$$
\text { - } \int \cdots \frac{1}{(2 \pi)^{k / 2}} \exp \left[-\frac{1}{2} \sum_{i=1}^{k} r_{i}^{2}\right] \prod_{i=1}^{k} d r_{i} \prod_{i=1}^{k} d s_{i} \cdot d \chi^{2}
$$$$
r_{k}<n_{k} / l_{k k}
$$$$
r_{k-1}<\left(n_{k-1}-l_{k-1, k} r_{k}\right) / l_{k-1, k-1}
$$$$
r_{1}<\left(n_{1}-{ }_{k} \sum_{j}=2 l_{1} r_{j}\right) / l_{11}
$$

In combination of (2.3) and (2.4), we shall reach (2.1) which was to be proved.

Theorem 2.2. The mean vector $E\{\boldsymbol{x}\}$ and the mean square error matrix M.S.E. $\{\overline{\boldsymbol{x}}\}$ of the estimate $\boldsymbol{x}$ are given by

$$
E\{\boldsymbol{x}\}=\boldsymbol{\mu}^{(1)}+\frac{N_{2}}{N_{1}+N_{2}}\left(\boldsymbol{\mu}^{(2)}-\boldsymbol{\mu}^{(1)}\right) \operatorname{Pr}\left\{D_{1}\right\}-\sqrt{\frac{N_{2}}{N_{1}\left(N_{1}+N_{2}\right)}} \boldsymbol{c} I_{D_{2}}\{\boldsymbol{p}\}
$$

and

$$
\begin{aligned}
\text { M.S.E. }\{\boldsymbol{x}\} & =\frac{1}{N_{1}+N_{2}} \boldsymbol{\Sigma}+\left(\frac{N_{2}}{N_{1}+N_{2}}\right)^{2}\left(\boldsymbol{\mu}^{(2)}-\boldsymbol{\mu}^{(1)}\right)\left(\boldsymbol{\mu}^{(2)}-\boldsymbol{\mu}^{(1)}\right)^{\prime} \operatorname{Pr} .\left\{D_{1}\right\} \\
& +\frac{N_{2}}{N_{1}} I_{D_{2}}\left\{\boldsymbol{F} \boldsymbol{p} \boldsymbol{p}^{\prime} \boldsymbol{F}^{\prime}\right\}
\end{aligned}
$$


where we put

$$
\begin{aligned}
\operatorname{Pr}_{.}\left\{D_{1}\right\}= & \int \cdots \int \frac{1}{(2 \pi)^{k / 2} \boldsymbol{R}^{1 / 2}} \exp \left[-\frac{1}{2} \boldsymbol{p}^{\prime} \boldsymbol{R}^{-1} \boldsymbol{p} f_{N_{1}+N_{2}-k-1}\left(\chi^{2}\right) \prod_{i=1}^{k} d p_{i} \cdot d \chi^{2}\right. \\
& {\left[\left\{(\boldsymbol{p}+\boldsymbol{a})^{\prime} \boldsymbol{R}^{-1}(\boldsymbol{p}+\boldsymbol{a})\right\} / \chi^{2}\right]\left[\left(N_{1}+N_{2}-k-1\right) / k\right] \leq F_{k, N_{1}+N_{2}-k-1}(\alpha) } \\
I_{D_{2}}\{\boldsymbol{p}\}= & \int_{\left[\left\{(\boldsymbol{p}+\boldsymbol{a})^{\prime} \boldsymbol{R}^{-1}(\boldsymbol{p}+\boldsymbol{a})\right\} / \chi^{2}\right]\left[\left(N_{1}+N_{2}-k-1\right) / k\right]>F_{k, N_{1}+N_{2}-k-1}(\alpha)} \frac{\boldsymbol{p}}{(2 \pi)^{k / 2}} \boldsymbol{R}^{1 / 2} \exp \left[-\frac{1}{2} \boldsymbol{p}^{\prime} \boldsymbol{R}^{-1} \boldsymbol{p}\right] f_{N_{1}+N_{2}-k-1}\left(\chi^{2}\right) \prod_{i=1}^{k} d p_{i} \cdot d \chi^{2}
\end{aligned}
$$

Proof. Making use of the notation in the enunciation and proof of Theorem 2.1, we may write

$$
\begin{aligned}
E\{\overline{\boldsymbol{x}}\} & =\int \ldots \int_{D_{1}}\left\{\boldsymbol{\mu}^{(1)}+\boldsymbol{F} \boldsymbol{q}+\frac{N_{2}}{N_{1}+N_{2}}\left(\boldsymbol{\mu}^{(2)}-\boldsymbol{\mu}^{(1)}\right)\left\{h\left(\boldsymbol{p}^{\prime}, \boldsymbol{q}^{\prime}\right) f_{N_{1}+N_{2}-k-1}\left(\chi^{2}\right) \prod_{i=1}^{k} d p_{i} \cdot \prod_{i=1}^{k} d q_{i} \cdot d \chi^{2}\right.\right. \\
& +\int \ldots \int_{D_{2}}\left(\boldsymbol{\mu}^{(1)}+\boldsymbol{F} \boldsymbol{q}-G \boldsymbol{F} \boldsymbol{p}\right) h\left(\boldsymbol{p}^{\prime}, \boldsymbol{q}^{\prime}\right) f_{N_{1}+N_{2}-k-1}\left(\chi^{2}\right) \prod_{i=1}^{k} d p_{i} \cdot \prod_{i=1}^{k} d q_{i} \cdot d \chi^{2} \\
& =\boldsymbol{\mu}^{(1)}+\boldsymbol{F} I_{D_{1}+D_{2}}\{\boldsymbol{q}\}+\frac{N_{2}}{N_{1}+N_{2}}\left(\boldsymbol{\mu}^{(2)}-\boldsymbol{\mu}^{(1)}\right) \operatorname{Pr} .\left\{D_{1}\right\}-G \boldsymbol{F} I_{D_{2}} \\
& =\boldsymbol{\mu}^{(1)}+\frac{N_{2}}{N_{1}+N_{2}}\left(\boldsymbol{\mu}^{(2)}-\boldsymbol{\mu}^{(1)}\right) \operatorname{Pr} .\left\{D_{1}\right\}-\sqrt{\frac{N_{2}}{N_{1}\left(N_{1}+N_{2}\right)}} \boldsymbol{c I} I_{D_{2}}\{\boldsymbol{p}\},
\end{aligned}
$$

because $I_{D_{1}+D_{2}}\{\boldsymbol{q}\}=E\{\boldsymbol{q}\}=0$. Thus the mean vector (2.5) is obtained. The mean square error matrix M.S.E. $\{\overline{\boldsymbol{x}}\}$ may be obtained from following relation:

$$
\begin{aligned}
& E\left\{\overline{\boldsymbol{x}} \overline{\boldsymbol{x}}^{\prime}\right\}=\int \ldots \int_{D_{1}} \boldsymbol{\mu}^{(1)}+\boldsymbol{F} \boldsymbol{q}+\frac{N_{2}}{N_{1}+N_{2}}\left(\boldsymbol{\mu}^{(2)}-\boldsymbol{\mu}^{(1)}\right)\left(\boldsymbol{\mu}^{(1)}+\boldsymbol{F} \boldsymbol{q}\right. \\
& \left.+\frac{N_{2}}{N_{1}+N_{2}}\left(\boldsymbol{\mu}^{(2)}-\boldsymbol{\mu}^{(1)}\right)\right)^{\prime} h\left(\boldsymbol{p}^{\prime}, \boldsymbol{q}^{\prime}\right) f_{N_{1}+N_{2}-k-1}\left(\chi^{2}\right) \prod_{i=1}^{k} d p_{i} \cdot \prod_{i=1}^{k} d q_{i} \cdot d \chi^{2} \\
& +\int \cdots \int_{D_{2}}\left(\boldsymbol{u}^{(1)}+\boldsymbol{F} \boldsymbol{q}-\boldsymbol{G F} \boldsymbol{p}\right)\left(\boldsymbol{\mu}^{(1)}+\boldsymbol{F} \boldsymbol{q}\right. \\
& -G \boldsymbol{F} \boldsymbol{p})^{\prime} h\left(\boldsymbol{p}^{\prime}, \boldsymbol{q}^{\prime}\right) f_{N_{1}+N_{2}-k-1}\left(\chi^{2}\right) \prod_{i=1}^{k} d p_{i} \cdot \prod_{i=1}^{k} d q_{i} \cdot d \chi^{2} \\
& =\int \ldots \int_{D_{1}}\left\{\boldsymbol{\mu}^{(1)} \boldsymbol{\mu}^{(1) \prime}+\boldsymbol{F} \boldsymbol{q} \boldsymbol{q}^{\prime} \boldsymbol{F}^{\prime}+\frac{N_{2}}{N_{1}+N_{2}}\left(\boldsymbol{\mu}^{(1)}\left(\boldsymbol{\mu}^{(2)}-\boldsymbol{\mu}^{(1)}\right)^{\prime}+\left(\boldsymbol{\mu}^{(2)}-\boldsymbol{\mu}^{(1)}\right) \boldsymbol{\mu}^{(1) \prime}\right)\right. \\
& +\left(\frac{N_{2}}{N_{1}+N_{2}}\right)^{2}\left(\boldsymbol{u}^{(2)}-\boldsymbol{\mu}^{(1)}\right)\left(\boldsymbol{u}^{(2)}-\boldsymbol{\mu}^{(1)}\right)^{\prime}+\left(\boldsymbol{u}^{(1)} \boldsymbol{q}^{\prime} \boldsymbol{F}^{\prime}+\boldsymbol{F} \boldsymbol{q} \boldsymbol{\mu}^{(1) \prime}\right) \\
& +\frac{N_{2}}{N_{1}+N_{2}}\left(\boldsymbol{F} \boldsymbol{q}\left(\boldsymbol{\mu}^{(2)}-\boldsymbol{\mu}^{(1)}\right)^{\prime}+\left(\boldsymbol{\mu}^{(2)}-\boldsymbol{\mu}^{(1)}\right) \boldsymbol{q}^{\prime} \boldsymbol{F}^{\prime}\right) h\left(\boldsymbol{p}, \boldsymbol{q}^{\prime}\right) f_{N_{1}+N_{2}-k-1}\left(\chi^{2}\right) \\
& \prod_{i=1}^{k} d p_{i} \cdot \prod_{i=1}^{k} d q_{i} \cdot d \chi^{2}
\end{aligned}
$$




$$
\begin{aligned}
& +\int \ldots \int_{J_{2}}\left\{\boldsymbol{\mu}^{(1)} \boldsymbol{\mu}^{(1) \prime}+\boldsymbol{F} \boldsymbol{q} \boldsymbol{q}^{\prime} \boldsymbol{F}^{\prime}+G^{2} \boldsymbol{F} \boldsymbol{p} \boldsymbol{p}^{\prime} \boldsymbol{F}^{\prime}+\left(\boldsymbol{\mu}^{(1)} \boldsymbol{q}^{\prime} \boldsymbol{F}^{\prime}+\boldsymbol{F} \boldsymbol{q} \boldsymbol{\mu}^{(1) \prime}\right)\right. \\
& \left.\quad-G\left(\boldsymbol{\mu}^{(1)} \boldsymbol{p}^{\prime} \boldsymbol{F}^{\prime}+\boldsymbol{F} \boldsymbol{p} \boldsymbol{\mu}^{(1) \prime}\right)-G\left(\boldsymbol{F} \boldsymbol{q} \boldsymbol{p}^{\prime} \boldsymbol{F}^{\prime}+\boldsymbol{F} \boldsymbol{p} \boldsymbol{q}^{\prime} \boldsymbol{F}^{\prime}\right)\right\} \boldsymbol{h}\left(\boldsymbol{p}^{\prime}, \boldsymbol{q}^{\prime}\right) f_{N_{1}+N_{2}-k-1}\left(\chi^{2}\right) \\
& \quad \cdot \prod_{i=1}^{k} d p_{i} \cdot \prod_{i=1}^{k} d q_{i} \cdot d \chi^{2}
\end{aligned}
$$

substituting the following relations

$$
\begin{aligned}
& I_{D_{1}+D_{2}}\left\{\boldsymbol{q} \boldsymbol{q}^{\prime}\right\}=\int_{D_{1}+D_{2}} \ldots \int_{\boldsymbol{q}} \boldsymbol{\boldsymbol { q } ^ { \prime }} \cdot h\left(\boldsymbol{p}^{\prime}, \boldsymbol{q}^{\prime}\right) f_{N_{1}+N_{2}-k-1}\left(\chi^{2}\right) \cdot \prod_{i=1}^{k} d p_{i} \cdot \prod_{i=1}^{k} d q_{i} \cdot d \chi^{2}=E\left\{\boldsymbol{q} \boldsymbol{q}^{\prime}\right\}=\boldsymbol{R}, \\
& I_{D_{1}+D_{2}}\{\boldsymbol{q}\}=\boldsymbol{I}_{D_{2}}\{\boldsymbol{q}\{=0, \\
& \boldsymbol{I}_{D_{2}}\left\{\boldsymbol{q} \boldsymbol{p}^{\prime}\right\}=I_{D_{2}}\left\{\boldsymbol{p} \boldsymbol{q}^{\prime}\right\}=0
\end{aligned}
$$

for (2.9), we may obtain that

$$
\begin{aligned}
E\left\{\overline{\bar{x}} \overline{\bar{x}}^{\prime}\right\} & =\boldsymbol{\mu}^{(1)} \boldsymbol{\mu}^{(1) \prime}+\frac{1}{N_{1}+N_{2}} \boldsymbol{\Sigma}+\frac{N_{2}}{N_{1}+N_{2}}\left\{\boldsymbol{\mu}^{(1)}\left(\boldsymbol{\mu}^{(2)}-\boldsymbol{\mu}^{(1)}\right)^{\prime}+\left(\boldsymbol{\mu}^{(2)}-\boldsymbol{\mu}^{(1)}\right) \boldsymbol{\mu}^{(1) \prime}\right\} \operatorname{Pr} .\left\{D_{1}\right\} \\
& +\left(\frac{N_{2}}{N_{1}+N_{2}}\right)^{2}\left(\boldsymbol{\mu}^{(2)}-\boldsymbol{\mu}^{(1)}\right)\left(\boldsymbol{\mu}^{(2)}-\boldsymbol{\mu}^{(1)}\right)^{\prime} \operatorname{Pr} .\left\{D_{1}\right\}+\frac{N_{2}}{N_{1}} I_{D_{2}}\left\{\boldsymbol{F} \boldsymbol{p} \boldsymbol{p}^{\prime} \boldsymbol{F}^{\prime}\right\} \\
& -\sqrt{\frac{N_{2}}{N_{1}}} I_{D_{2}}\left\{\boldsymbol{\mu}^{(2)} \boldsymbol{p}^{\prime} \boldsymbol{F}^{\prime}+\boldsymbol{F} \boldsymbol{p} \boldsymbol{\mu}^{(1) \prime}\right\} .
\end{aligned}
$$

Thus the mean square error matrix M.S.E. $\{\bar{x}\}$ of the estimate $\bar{x}$ may be obtained in the following manner.

$$
\begin{aligned}
M . S . E .\{\overline{\boldsymbol{x}}\} & =E\left\{\left(\overline{\boldsymbol{x}}-\boldsymbol{\mu}^{(1)}\right)\left(\overline{\boldsymbol{x}}-\boldsymbol{\mu}^{(1)}\right)^{\prime}\right\} \\
& =E\left\{\boldsymbol{x} \overline{\boldsymbol{x}}^{\prime}\right\}-\boldsymbol{\mu}^{(1)} E\left\{\overline{\boldsymbol{x}}^{\prime}\right\}-E\{\overline{\boldsymbol{x}}\} \boldsymbol{\mu}^{(1) \prime}+\boldsymbol{\mu}^{(1)} \boldsymbol{\mu}^{(1) \prime} \\
& =\frac{1}{N_{1}+N_{2}} \boldsymbol{\Sigma}+\left(\frac{N_{2}}{N_{1}+N_{2}}\right)^{2}\left(\boldsymbol{\mu}^{(2)} \boldsymbol{\mu}^{(1)}\right)\left(\boldsymbol{\mu}^{(2)}-\boldsymbol{\mu}^{(1)}\right)^{\prime} \operatorname{Pr} .\left\{D_{1}\right\} \\
& +\frac{N_{2}}{N_{1}} I_{D_{2}}\left\{\boldsymbol{F} \boldsymbol{p} \boldsymbol{p}^{\prime} \boldsymbol{F}^{\prime}\right\} .
\end{aligned}
$$

Corollary 2.1. Specially under the same assumption as corollary 1.1 or corollary 1.2, the same results are obtained correspondently.

Corollary 2.2. Specially when $\mu^{(2)}=\mu^{(1)}$, the variance component of the M.S.E. $\{\boldsymbol{x}\}$ in case of multivariate pooling of data becomes smaller than the variance of the corresponding estimate in case of univariate pooling of data. The difference is given by

$$
\frac{N_{2} \sigma_{i i}}{\left(N_{1}+N_{2}\right) N_{1}}\left[I_{D_{2}}\left\{p_{i}^{2}\right\}-I_{D_{2 *}}\left\{p_{i}^{2}\right\}\right]<0 \quad(i=1,2, \cdots, k)
$$

where 


$$
\begin{aligned}
I_{D_{2}}\left\{p_{i}^{2}\right\}= & \iint_{D \cdot 2} p_{i}^{2} \cdot \frac{1}{(2 \pi)^{k / 2}} \overline{R^{1 / 2}} \exp \left[-\frac{1}{2} \boldsymbol{p}^{\prime} \boldsymbol{R}^{-1} \boldsymbol{p}\right] f_{N_{1}+x_{2}-k-1}\left(\chi^{2}\right) \cdot \prod_{i=1}^{k} d p_{i} \cdot d \chi^{2} \\
I_{D^{2}}\left\{p_{i}^{2}\right\}= & \iint p_{i}^{2} \cdot \frac{1}{(2 \pi)^{1 / 2}} \exp \left[-\frac{1}{2} p_{i}^{2}\right] f_{N_{1}+N_{2}-k-1}\left(\chi^{2}\right) d p_{i} d \chi^{2} \\
& \frac{\left(N_{1}+N_{2}-2\right)^{1 / 2} p_{i}+\left(\frac{N_{1} N_{2}}{N_{1}+N_{2}}\right)^{1 / 2} \frac{\mu_{i}(2)-\mu_{i}(1)}{\sigma}}{\left(\chi^{2}\right)^{1 / 2}}>t_{N_{1}+x_{2}-2}(\alpha)
\end{aligned}
$$

\section{References}

Asano, C. [1] : Estimations after preliminary test of significance and their applications to biometrical researches, Bull. Math. Stat., 9-1, 2 (1960), 1-23.

Asavo, C. and Sato, S. [1] : A bivariate analogue of pooling data, Bull. Math. Stat. 11 (1962), (in preparation).

Bancroft, T. A. [1] : On biases in estimation due to the use of preliminary tests of significance, Ann. Math. Stat., 15 (1944), 190-204.

BENNET, C. I. [1] : Estimation of means on the basis of preliminary tests of signiflcance, Ann. Inst. Stat. Math., Tokyo, 4 (1952), 31-43.

Kitagawa, T. [1] : Successive process of statistical inferences, Mem. Facu. of Sci. Kyushu Univ., Ser. A, 5 (1950), 139-180.

Kitagawa, T. [2]: Successive process of statistical inferences, (in Japanese, Suisoku katei Ron), Iwauami, Tokyo, 1958.

SAGA UNIVERSITY 